Next time, I would survey paediatric doctors and ENPs in addition to emergency care doctors as they also complete the checklist while clerking.

Message for others Without regular measurements, we could not tell if the checklist was being used. To find out it is being used poorly through a serious case review is not acceptable. By regular measurement, we have weekly data which provides more detailed information on completion rates. Staff engagement was vital to improving the checklist and increasing compliance, as detailed above.

\section{G581(P) IMPLEMENTATION OF A PAEDIATRIC PRESCRIBING POCKETGUIDE}

PAC Stilwell. Intensive Care, Royal Brompton Hospital, London, UK

\subsection{6/archdischild-2015-308599.530}

Context Neonatology department in a busy district general hospital.

Problem The project set out to address inpatient prescription errors which could result in illegal prescriptions and patient harm.

Assessment of problem and analysis of its causes I collected data from 20 random drug charts and used a checklist to identify the incidence and types of prescribing errors on in-patient drug charts for patients on Special Care Baby Unit and Neonatal Intensive care Unit. After completing the data collection, I met with paediatric pharmacists and departmental doctors and nurses to determine why certain prescribing erros were being made.

Intervention I wrote, designed and developed a pedaitric prescribing pocket guide to clarify local and national guidance regarding paediatric prescribing. I used my own drawings to make a user-friendly guide.

Study design Audit.

Strategy for change I worked with management and senior staff to get the paediatric prescribing guide approved and authorised. It has subsequently been published on the hospital intranet and is available as hardcopies in clinical areas. I used email and departmental meetings to advertise the new prescribing guide, as well as educating new arrivals about paediatric prescribing.

Measurement of improvement To measure the effects of my intervention, I re-audited the incidence and types of prescribing errors on in-patient drug charts. I compared the overall mean prescribing error rate, as well as specific prescribing errors.

Effects of changes Re-audit showed a reduction in mean prescribing error rate, although there is still room for improvement. This has led to clearer prescriptions and fewer uncertainties surrounding delivery of medication. Subjective feedback has reported that the guide has improved communication between multi-disciplinary team members reviewing and using drug charts.

Lessons learnt This project has taught me the need to collect feedback from different team members (pharmacists, nurses, doctors, specialist teams) to identify the reasons behind prescribing errors. I have also learnt how to develop new guidelines and the steps that are needed to go through in order to get new guielines approved and disseminated.

Message for others It is important to involve senior members of staff when developing new guidelines and to be persistent, yet realistic when it comes to implementing new guidelines e.g. printing the guidelines for all new arrivals is expensive whereas making the guidelines available on the trust intranet is cheap and accessible to all healthcare staff.

\section{G582(P) OPERATIVE NOTE QUALITY IMPROVEMENT - EXAMINING COMPLIANCE WITH THE ROYAL COLLEGE OF SURGEONS OF ENGLAND GUIDELINES IN A PAEDIATRIC GENERAL SURGICAL UNIT}

A Ryan, T Reeves, K Kuti, M Flett. Paediatric General Surgery, Royal Hospital for Sick Children Yorkhill, Glasgow, UK

\subsection{6/archdischild-2015-308599.531}

Context Operative notes should be documented "clearly, accurately and legibly" - as endorsed by the GMC's Good Medical Practice. The RCS Eng. Guidelines (2014) advocate contemporaneous, complete surgical records. This audit identified significant shortcomings in operative documentation amongst paediatric general surgical cases in a tertiary care centre. Data collection was performed by two FY1s and a Surgical Registrar.

Problem Surgical operation notes necessitate accurate documentation from both a medico-legal and professional perspective; whilst comprehensive records facilitate post-operative patient management. However; in practice they are frequently substandard, thus raising concerns towards patient safety. Current operative note templates within our unit fail to comply with relevant guidelines.

Assessment of problem and analysis of its causes We identified poorly-documented details within general surgical operation notes using a data collection tool incorporating pertinent, recently-published guidance. Areas for improvement included: patient's weight, type of procedure performed (i.e. emergency vs. elective), whether surgery was with or without complication, and antibiotic prophylaxis. Results attained were compared against data from previous audits of a similar nature (carried out in 2010 and 2011 respectively).

Intervention Our proposed intervention involves promoting typed documentation of operative notes, whilst advocating their transition from paper text to an electronic format. We plan to achieve this through usage of a unique, pre-existing computerised system developed by a consultant within the department, which additionally populates our current Trakcare system upon document creation. We hope to educate the entire general surgical team in its practice.

Furthermore, we will introduce informative posters highlighting the RCS Eng. guidelines and reiterating imperative operative details within the main theatres and day surgery unit. Letters detailing the audit's key findings and our intended strategy for change were sent to all consultants and surgical trainees.

Study design Prospective analysis of 138 general surgical operative notes identified over a 2 -week period. We created a 26 point core checklist incorporating both RCS (Eng.) 2014 and GMC "Good Medical Practice" 2013 guidance. We then audited elective and emergency operative documentation against this standardised proforma. Data collected was subsequently evaluated once compiled onto a computerised spreadsheet (Table 1). Strategy for change Our findings were presented at the weekly departmental meeting, where we emphasised the need for operative note improvement. Proposed interventions were then implemented at the earliest opportunity. In particular, details not previously recorded, yet strongly advocated, by the RCS Eng. were promoted (e.g. anticipated blood loss, DVT prophylaxis etc.). 


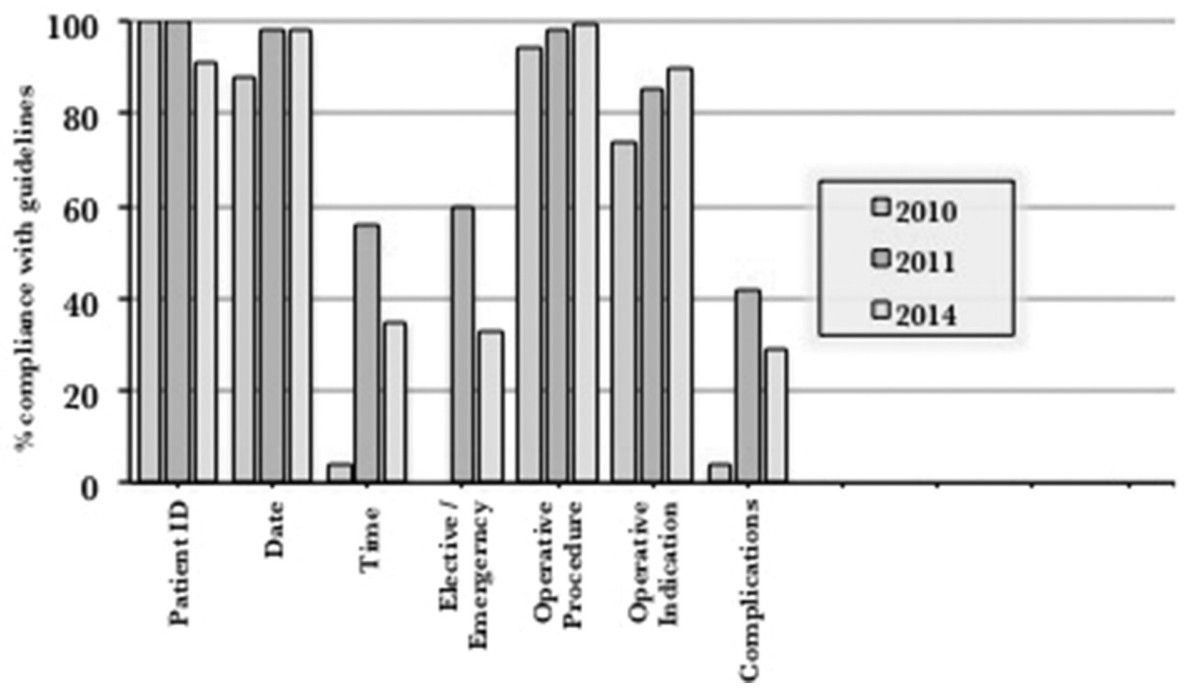

Measurement of improvement We intend to re-audit operation notes in 2-3 months' time, using an identical proforma to record data, once the proposed interventions have been implemented. We will determine whether results attained are statistically significant by calculating $\mathrm{p}$-values and using simple statistical data analysis.

Effects of changes We anticipate enhanced operative note standards as a result of introducing the proposed interventions. Our aspiration is to generate discussion in computerised documentation of surgeries and inspire greater numbers of surgeons to make use of electronic systems to ensure operative notes are both standardised and increasingly legible.

Lessons learnt Surgeons were receptive to this study's findings and were genuinely keen to improve upon their own operative documentation. Concerns were raised as to whether certain areas of documentation advised by the RCS Eng. are applicable to the paediatric populace e.g. DVT prophylaxis. This highlighted the importance of considering your patient cohort when formulating an operative note template.

Message for others We envisage the future of operative notes to be an electronic format. However, until this is achieved we recognise that the majority of surgeons will continue to use our current paper-based arrangement. Thus, we believe that typed operative documentation (using the methods described above) is an acceptable platform towards ameliorating the existing Trakcare system.

\section{G583(P) IMPROVING INTERDISCIPLINARY COMMUNICATION ON A POSTNATAL WARD}

C Duncan, E Sturdy, D Armstrong. Paediatric Department, Altnagelvin Area Hospital, Derry, UK

\subsection{6/archdischild-2015-308599.532}

Context Our hospital serves a surrounding population of over two hundred and fifty thousand people and caters to about three thousand deliveries a year. There is one postnatal ward with nineteen beds for mothers and their babies. It is usually staffed by three midwives, two healthcare assistants and one paediatric SHO during working hours.
Problem Communication between the obstetric, midwifery and paediatric staff regarding any babies on the postnatal ward was casual and ad hoc with no set verbal or written handover between daytime and out of hours staff. This appeared to lead to delays in treatment of certain babies, for instance antibiotic administration, screening for sepsis and serum bilirubin measurements.

Assessment of problem and analysis of its causes A multidisciplinary survey of those involved in obstetric and neonatal care was undertaken to identify the cause of these problems and to garner suggestions for improvement.

Intervention A more formalised handover system was developed. Information was passed between the daytime paediatric SHO to the on call team using a printed handover sheet passed from the daytime paediatric SHO to the on call team. The sheet contains a list of babies needing specific paediatric input and includes aide memoires of NICE guidelines concerning early onset neonatal sepsis and neonatal jaundice. At change of shifts patients are discussed with the written sheet as a reminder.

Study design This study was carried out as a clinical practice improvement project.

Strategy for change The printed handover sheet was introduced on a Monday morning for a trial of four weeks. It was used at each morning, early evening and night-time handover. Its introduction followed discussion with the paediatric medical staff and the midwifery team via the ward sister.

Measurement of improvement The staff of both departments were surveyed a month later to feed back any changes brought about through the new handover system. The results were very encouraging with $100 \%$ respondents reporting an improvement in communication of postnatal duties.

Effects of changes There was a improvement in both the speed at which babies were reviewed and management plans instigated. The number of bleeps to the postnatal SHO decreased. No problems were encountered throughout the period of quality improvement. The midwifery staff are now considering introducing a similar system for their daily handover.

Lessons learnt Importance of asking for feedback from all staff involved on the ward to obtain opinions on the need for change was the best way to do this. It has ensured any improvement introduced has been user friendly and staff are enthusiastic with regards to the change. 